\title{
Cysteine Scanning Mutagenesis of TM4b-4c Loop of Glutamate Transporter EAAT1 Reveals Three Conformationally Sensitive Residues $\mathbf{s}$
}

\author{
Wenlong Zhang, Xiuping Zhang, and Shaogang Qu
}

Clinical Medicine Research Centre (W.Z., S.Q.) and Department of Neurology (W.Z., S.Q.), Shunde Hospital, Southern Medical University, Foshan, Guangdong, People's Republic of China; and Teaching Center of Experimental Medicine, School of Basic Medical Sciences, Southern Medical University, Guangzhou, Guangdong, People's Republic of China (X.Z.)

Received November 25, 2017; accepted April 11, 2018

\section{ABSTRACT}

Glutamatergic synaptic transmitters are cleared from the synaptic cleft through excitatory amino acid transporters (EAATs) that are responsible for recycling glutamate and transporting it into neurons and glial cells. To probe the structural role of the TM4b-4c loop of EAAT1 (Rattus norvegicus), each of the 57 amino acid residues was mutated to cysteine. Thirteen of the single mutants have very low transport activity. Aqueous accessibility of the introduced cysteines from the remaining mutants was then explored by membranepermeant and membrane-impermeant sulfhydryl reagents in different conditions. F190C, V238C, and A243C were affected by MTSET, whereas Q189C, F190C, V238C, A243C, and L244C were sensitive to MTSEA. Q189C and L244C transport activity was diminished in the presence of potassium, which is expected to favor the inward-facing conformation of the transporter. Inversely, L244C was protected by glutamate. The modification of A243C by MTSEA was enhanced by either potassium and glutamate or DL-threo- $\beta$-benzyloxyaspartate. From these results, we suggest that residues F190C, V238C, and A243C may be located near the extracellular surface, and the TM4b-4c loop forms multiple reentrant membrane loops on the cell surface. Alternatively, F190C, V238C, and A243C may function in the transport pathway, which is exposed to MTSET. In addition, Q189C, A243C, and L244C are conformationally sensitive and may play a role in the transport cycle.

\section{Introduction}

Glutamate is the predominant excitatory transmitter in the central nervous system. To prevent hyperexcitability and neurotoxicity, it is recycled from the synapse cleft by excitatory amino acid transporters (EAATs) sited in the surface membrane of neurons and glial cells (Kanner and Schuldiner, 1987; Nicholls and Attwell, 1990; Rothstein et al., 1996; Zerangue and Kavanaugh, 1996; Tanaka et al., 1997; Levy et al., 1998). Glutamate transporters play an important role in terminating synaptic excitation (Tong and Jahr, 1994; Otis et al., 1996; Diamond and Jahr, 1997; Verdon et al., 2014). Glutamate transport is an electrogenic translocation generated by the $\mathrm{Na}^{+} / \mathrm{K}^{+}$-ATPase (Kanner and Sharon, 1978; Brew and Attwell, 1987; Wadiche et al., 1995). To maintain steep transmembrane glutamate gradients, EAATs transport one substrate molecule in symport with three sodium ions and one proton. In the second half-cycle, one potassium ion is released

This work was supported by grants from the National Natural Science Foundation of China [Grant 31570716, U1603281]. No potential conflicts of interest relevant to this article are reported.

https://doi.org/10.1124/mol.117.111245.

S This article has supplemental material available at molpharm.aspetjournals.org. to the extracellular space and the next cycle of transporter starts (Zerangue and Kavanaugh, 1996; Levy et al., 1998; Owe et al., 2006). There are five known subtypes of EAATs: EAAT1, GLT-1, excitatory amino acid carrier-1, EAAT4, and EAAT5. Also, they share about amino acid identity of $50 \%$ (Kanai and Hediger, 1992; Pines et al., 1992; Storck et al., 1992; Arriza et al., 1994, 1997; Fairman et al., 1995).

The crystal structures of aspartate transporter from the archaeon glutamate transporter homolog from Pyrococcus horikoshii $\left(\mathrm{Glt}_{\mathrm{Ph}}\right)$ have advanced our understanding of eukaryotic glutamate transporters and provided insights into various aspects of transport, in accordance with biochemical, electrophysiological, and other experimental data (Yernool et al., 2004; Boudker et al., 2007; Kanner, 2007; Crisman et al., 2009; Reyes et al., 2009; Teichman et al., 2009; Verdon and Boudker, 2012). The structure illuminates a trimer with a permeation pathway through each of the monomers, suggesting that the monomer is the fully independent and functional unit. The same is true for the eukaryotic glutamate transporters (Grewer et al., 2005; Koch and Larsson, 2005; Koch et al., 2007a; Leary et al., 2007). The functional unit is a monomer containing eight transmembrane domain (TM) helices (TM1 TM8) and two

ABBREVIATIONS: $\mathrm{ChCl}$, choline chloride; CL-EAAT1, cysteine-less excitatory amino acid transporter 1 ; DL-TBOA, DL-threo- $\beta$-benzyloxyaspartate; EAAT, excitatory amino acid transporter; FRET, fluorescence resonance energy transfer; GLT-1, glial glutamate transporter type 1; Gltph, glutamate transporter homolog from Pyrococcus horikoshii; HP, hairpin loop; MTSEA, 2-aminoethyl methanethiosulfonate; MTSET, (2-trimethylammonium)ethyl methanethiosulfonate; TM, transmembrane domain. 
oppositely oriented re-entrant loops (HP1 and HP2) (Boudker et al., 2007; Shrivastava et al., 2008; Reyes et al., 2009; Canul-Tec et al., 2017). HP1 and HP2 are located on opposite sides of the cell membrane. HP2 participates in the extracellular gating for the substrate reaction, whereas HP1 has been postulated to form the intracellular gate (Yernool et al., 2004; Huang and Tajkhorshid, 2008; Shrivastava et al., 2008; Crisman et al., 2009; Reyes et al., 2009). The amino acid insertions and deletions, together with the differences on the transport function and pharmacology of $\mathrm{Glt}_{\mathrm{Ph}}$, make it impossible to uncover the structural mechanism of the EAATs. The TM4 domain in EAATs is composed of three separate helices-4a, 4b, and 4c-with Glt ${ }_{\mathrm{Ph}}$ sharing about $35 \%$ sequence identity with EAAT1 (Fig. 1). The latter has 57 more amino acid residues located in the TM4b-4c loop (Yernool et al., 2004). To study the molecular and structural determinants in glutamate transport, we introduced a cysteine residue to replace each of the amino acids within the TM4b-4c loop of a cysteine-less EAAT1 (CL-EAAT1) (Seal and Amara, 1998). Membrane-permeant (2-trimethylammonium)ethyl methanethiosulfonate (MTSET) and membrane-impermeant 2-aminoethyl methanethiosulfonate (MTSEA) sulfhydryl reagents were used to explore the aqueous accessibility of introduced cysteines under conditions of either inwardfacing or outward-facing conformations. Residues F190C, V238C, and A243C were sensitive to MTSET. These results suggest that residues in the TM4b-4c loop domain may participate in forming an aqueous transport pathway or may be located at the side, with Q189, A243, and L244 directly involved in the transport cycle.

\section{Materials and Methods}

Generation of Mutants. CL-EAAT1 in the vector pBluescript SK (-) (Stratagene, La Jolla, CA) was used as the parent template for site-directed mutagenesis. Mutant transporters were generated using the QuikChange Site-Directed Mutagenesis Kit (TOYOBO, Osaka, Japan). All mutant sequences were confirmed by full-length sequencing (Invitrogen, Waltham, MA).

Cell Growth and Expression of Transporter. HeLa cells purchased from the American Type Culture Collection (Manassas, VA) were cultured in Dulbecco's modified Eagle's medium basic $1 \times$ (Invitrogen) with $8 \%$ fetal calf serum (Invitrogen), $200 \mathrm{U} / \mathrm{ml}$ penicillin (Beyotime Biotechnology, Shanghai, People's Republic of China), and $200 \mu \mathrm{g} / \mathrm{ml}$ streptomycin (Beyotime Biotechnology).
Infections with recombinant vaccinia/T7 virus in serum-free Dulbecco's modified Eagle's medium basic $1 \times$ were carried out for 30 minutes (Fuerst et al., 1986). CL-EAAT1 or mutant constructs were then added into the virus-infected HeLa cells with Lipo6000 Transfection Reagent (Beyotime Biotechnology) and incubated at $37^{\circ} \mathrm{C}$ and $5 \% \mathrm{CO}_{2}$ for 20 hours.

Transport Assay. Cells that expressed mutants in 24-well plates were washed with choline chloride $(\mathrm{ChCl})$ solution $(5 \mathrm{mM}$ $\mathrm{KPi}, \mathrm{pH} 7.4,150 \mathrm{mM} \mathrm{ChCl}, 0.5 \mathrm{mM} \mathrm{MgSO}_{4}$, and $0.3 \mathrm{mM} \mathrm{CaCl}_{2}$ ) and incubated with $\mathrm{NaCl}$ solution (5 mM KPi, pH 7.4, $150 \mathrm{mM}$ $\left.\mathrm{NaCl}, 0.5 \mathrm{mM} \mathrm{MgSO}_{4}, 0.3 \mathrm{mM} \mathrm{CaCl}_{2}\right)$ containing $0.4 \mu \mathrm{Ci}(0.15 \mu \mathrm{M})$ D- $\left[{ }^{3} \mathrm{H}\right]$-aspartate (PerkinElmer, Waltham, MA) for 10 minutes. The reaction was stopped using ice-cold $\mathrm{NaCl}$ solution and lysed with $1 \%$ sodium dodecyl sulfate. Finally, radioactivity was assayed (Fuerst et al., 1986).

Inhibition Studies with Sulfhydryl Reagents. Cells that expressed mutants in 24-well plates were washed once with $\mathrm{ChCl}$ solution. $\mathrm{NaCl}$ solution containing varying concentrations of MTSET (Biotium, Fremont, CA) or MTSEA (Biotium) were incubated at $37^{\circ} \mathrm{C}$ for 5 minutes. Cells were then washed twice with $\mathrm{NaCl}$ solution and transport activity was subsequently measured. The concentrations of the sulfhydryl reagents were optimized for each mutant to show a partial inhibition.

Effects of Transporter Substrates and Inhibitors on Sulfhydryl Modification. The following reagents were each supplemented to the preincubation media, along with MTSET or MTSEA, for the following inhibition assays: $150 \mathrm{mM} \mathrm{NaCl}$ solution, and $150 \mathrm{mM}$ $\mathrm{NaCl}$ solution $+1 \mathrm{mM}$ L-glutamate, $150 \mathrm{mM} \mathrm{NaCl}$ solution $+20 \mu \mathrm{M}$ DL-TBOA (Tocris Bioscience, Bristol, UK), $150 \mathrm{mM} \mathrm{KCl}$ solution, and $150 \mathrm{mM} \mathrm{ChCl}$ solution.

Cell Surface Biotinylation. HeLa cells that expressed CL-EAAT1 and the mutants $\mathrm{A} 243 \mathrm{C}$ and $\mathrm{L} 244 \mathrm{C}$ were washed in a 6-cm dish with icecold phosphate-buffered saline ( $\mathrm{pH}$ 8.0) after transfection for 24 hours. The cells were then incubated with ice-cold phosphate-buffered saline containing $0.5 \mathrm{mg} / \mathrm{ml}$ EZ-Link Sulfo-NHS-SS-Biotion (Thermo Fisher Scientific, Waltham, MA) for 20 minutes with gentle shaking. The reaction was stopped with $100 \mathrm{mM}$ glycine. The cells were then lysed on ice for 20 minutes with cell lysis buffer (Beyotime Biotechnology). The cell debris was separated by centrifugation at $12,000 \mathrm{rpm}$ for 20 minutes at $4^{\circ} \mathrm{C}$. Pierce Streptavidin Agarose (Thermo Fisher Scientific) beads were added to the supernatant and incubated for 40 minutes. The beads were then separated by centrifugation $\left(1\right.$ minute at $\left.4^{\circ} \mathrm{C}\right)$ and washed three times with ice-cold buffer, collecting the supernatants from each step into fresh tubes. Biotinylated proteins were separated from the streptavidin agarose beads by heat denaturation. Supernatants collected from each centrifugation step were subsequently analyzed using Western blots (Fuerst et al., 1986; Rong et al., 2016).

Western Blot Analysis. Protein samples were incubated in protein loading buffer at $55^{\circ} \mathrm{C}$ for 30 minutes prior to separation by

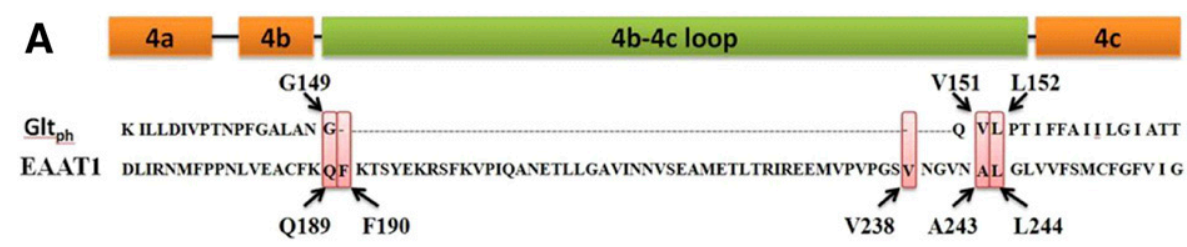

B

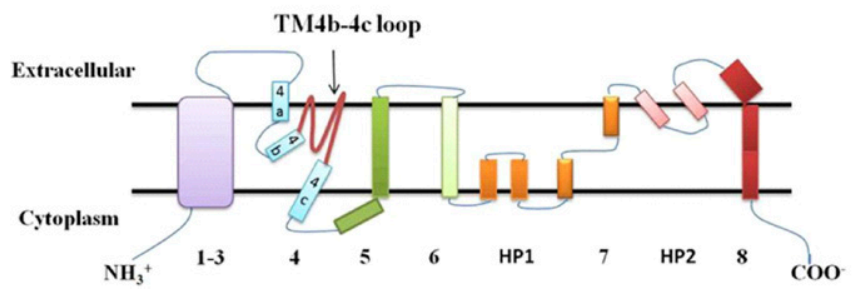

Fig. 1. Sequence alignment and EAAT transmembrane topology. (A) Sequence alignment of TM4b-4c loop on Glt ${ }_{\mathrm{Ph}}$ and EAAT1 (Yernool et al., 2004; Canul-Tec et al., 2017). Insertions in eukaryotic transporters between helices $4 \mathrm{~b}$ and $4 \mathrm{c}$ are not included and are marked by "Arrows represent residues Q189, F190, V238, A243, and L244 on EAAT1, and G149, V151, and L152 on Glt $_{\mathrm{Ph}}$. (B) Representation of EAAT transmembrane topology (Yernool et al., 2004). Putative position of the $4 b-4 c$ loop is depicted by the red loop. Arrows represent the TM4b-4c loop. 
SDS-PAGE and transfer onto polyvinylidene difluoride membranes. The blots were then treated with $5 \%$ bovine serum albumin for 1.5 hours at room temperature, and probed with anti-EAAT 1 antibody (Santa Cruz Biotechnology, Dallas, TX) for 16 hours at $4^{\circ} \mathrm{C}$. The membranes were washed three times with $1 \times$ Tris-buffered saline/Tween 20 and then incubated with the secondary antibody donkey anti-goat (Beyotime Biotechnology) for 1 hour at room temperature. Protein bands were visualized by chemiluminescence (Beyotime Biotechnology) and analyzed using ImageJ software. Expression levels for the biotinylated, nonbiotinylated, and total proteins in all samples were normalized to integrin (Santa Cruz Biotechnology), $\alpha / \beta$-tubulin (Cell Signaling Technology, Danvers, MA), and actin (Beyotime Biotechnology), respectively. $\left[{ }^{3} \mathrm{H}\right]$-Labeled uptake activity was normalized to relative cell surface expression (Hussainzada et al., 2006, 2008; Rong et al., 2016).

SWISS-MODEL. SWISS-MODEL is available at http://swissmodel. expasy.org/. The target sequence for Rattus norvegicus EAAT1 (GenBank accession number X63744.1; UniProtKB P24942) was uploaded for the modeling project. The template we used was the thermostable human EAAT1 (PDB code 5llm.1.A, Supplemental Material). The detailed report of the modeling is shown in Supplemental Material, and the SWISS-MODEL is available in Supplemental Material.

Data Analysis. At least three different experiments were performed, and data are shown as the mean \pm S.E. Statistics and analyses were conducted using SPSS version 20.0 statistical software using one-way analysis of variance. Data were considered statistically significant at $P<0.05$.

\section{Results}

Cysteine Scanning and Transport Activity of Single Cysteine Mutants. Fifty-seven single cysteine mutants were generated by replacing each of the amino acid residues within the TM4b-4c loop of CL-EAAT1 to a cysteine residue. EAAT1, CL-EAAT1, and the mutants were expressed in HeLa cells, and the uptake of $\mathrm{D}-\left[{ }^{3} \mathrm{H}\right]$-aspartate was measured and compared with that of CL-EAAT1. The uptake ability of the cysteine mutants ranged from $1.5 \% \pm 0.8 \%$ to $127.7 \% \pm$ $10.2 \%$ relative to CL-EAAT1. Thirteen mutants (K191C, T192C, Y194C, R197C, N206C, N216C, S218C, E230C, V232C, P233C, P235C, N242C, and G245C) had transport activities less than $15 \%$. Transport activity of these mutants ranged from $1.5 \% \pm 0.8 \%$ to $14.8 \% \pm 6.1 \%$ of the CL-EAAT1 (Fig. 2).
Accessibility of Single Cysteine Mutants. To probe for sulfhydryl modifications on the extracellular side of the bilayer, HeLa cells expressing either CL-EAAT1 or each of the 44 single cysteine mutants demonstrating measurable transport activity were treated with membrane-impermeant sulfhydryl reagent MTSET at $2.0 \mathrm{mM}$. Transport activities of F190C, V238C, and A243C were inhibited by MTSET (Fig. 3A) in a concentration-related way, with full inhibition of activity observed at $3.0 \mathrm{mM}$ MTSET (Fig. 3B). The others were not impacted by MTSET. Additionally, the cells were exposed to membrane-permeant MTSEA at $2.5 \mathrm{mM}$ to investigate the sulfhydryl modifications on the cytoplasmic side of the bilayer (Fig. 4A). Significant inhibition of the uptake activity of Q189C was detected (Fig. 4, A and C), whereas less inhibition was seen in F190C, V238C, A243C, and L244C (Fig. 4, A and B). Transport activity of these mutants decreased gradually with increasing MTSEA concentration (Fig. 4, B and C).

Effect of Transporter Substrates and Inhibitors on Sulfhydryl Modification in Single Cysteine Mutants. To increase exposure of the mutated residues to sulfhydryl modification, the cells were preincubated in media containing transporter substrates or inhibitors. Transport activities were measured on HeLa cells expressing Q189C, F190C, V238C, $\mathrm{A} 243 \mathrm{C}$, and $\mathrm{L} 244 \mathrm{C}$ that were preincubated in the presence of the sulfhydryl reagent with saturating amounts of transporter substrates or inhibitors. Transporter substrates or inhibitors did not affect sulfhydryl modification of F190C and V238C by MTSET (Fig. 5) and MTSEA (Fig. 6, B and C). Potassium slightly enhanced MTSEA modification in Q189C and L244C, whereas DL-TBOA did not show a significant effect (Fig. 6, A and E). However, glutamate protected L244C from MTSEA modification (Fig. 6E). Last, transporter substrates or inhibitors enhanced MTSEA modification of A243C (Fig. 6D).

Expression of Single Mutants A243C and L244C. To clarify the association between decreased transport activity and membrane expression of $\mathrm{A} 243 \mathrm{C}$ and L244C that may take part during the transport cycle, surface biotinylation was performed. Cell membrane expression of A243C and L244C was detected by membrane-impermeable biotin labeling and immunoblotting (Fig. 7A). The total proteins and the nonbiotinylated proteins of A243C and L244C showed no significant differences with CL-EAAT1 (Fig. 7, B and D). However,

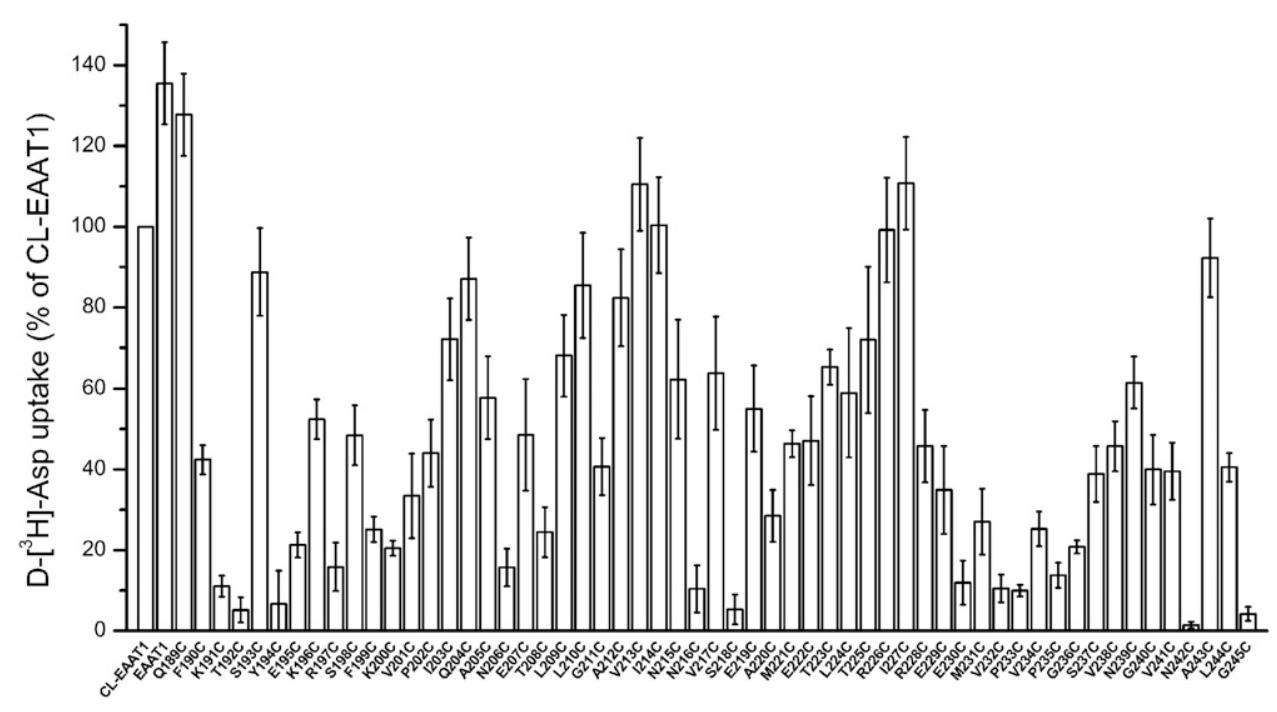

Fig. 2. Transport activity of single cysteine mutants of the TM4b-4c loop of CL-EAAT1. HeLa cells expressing single cysteine mutants, CL-EAAT1, and EAAT1 were washed twice with ChCl-containing solution, and subsequently $\mathrm{D}-\left[{ }^{3} \mathrm{H}\right]$-aspartate uptake was assayed. Data are given as a percentage of CL-EAAT1 transport activity and are the mean \pm S.E. of three to four experiments, each of which was performed in triplicate. 

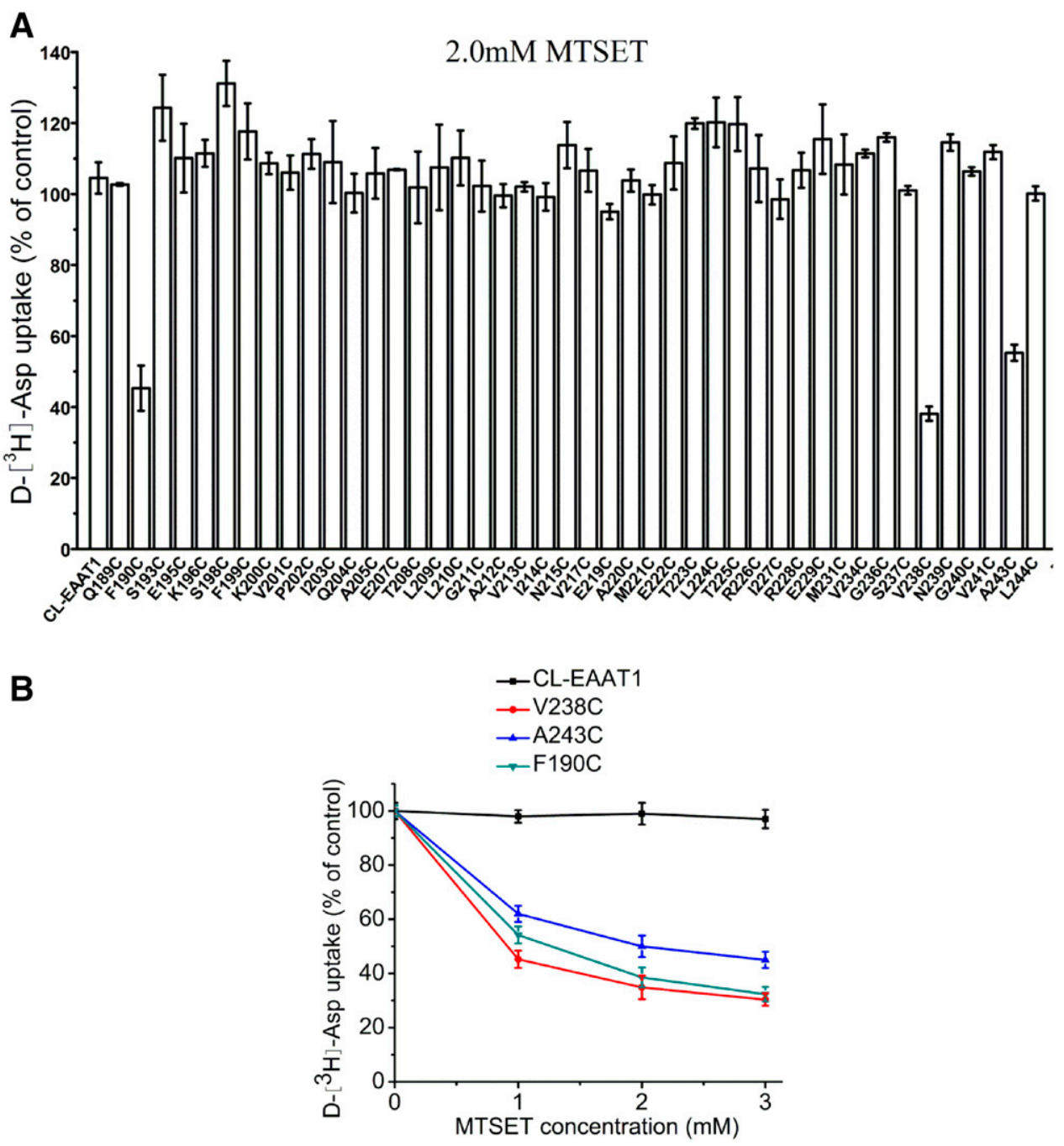

Fig. 3. Effect of MTSET on uptake activity of single cysteine mutants of the TM4b-4c loop of CL-EAAT1. HeLa cells expressing single cysteine mutants or CL-EAAT1 were preincubated in $\mathrm{NaCl}$-containing medium with MTSET for 5 minutes at room temperature and were washed twice with ChCl-containing solution; subsequently $\mathrm{D}-\left[{ }^{3} \mathrm{H}\right]$-aspartate transport was assayed. Data represent the percentage of the remaining uptake activity after incubation with MTSET relative to values obtained in the absence of MTSET and represent the mean \pm S.E. of at least three different experiments performed in triplicate. (A) Effect of MTSET on transport activity of single cysteine mutants of the TM4b-4c loop of CL-EAAT1. The concentration of MTSET used in this study was $2.0 \mathrm{mM}$. (B) Effect of different concentrations of MTSET on transport activity of F190C, V238C, and A243C.

there was a pronounced decline in the cell surface expression of L244C (Fig. 7C). Compared with CL-EAAT1, the ratio of biotinylated proteins to nonbiotinylated proteins dramatically decreased in L244C but not in A243C (Fig. 7E). Comparable to the cysteine-less parent, transport activity normalized to relative plasma membrane expression of A243C and L244C decreased (Fig. 7F).

\section{Discussion}

Our previous results have demonstrated that the glutamate transporter EAAT1 may experience complicated movement between TM4 and HP1 or HP2 in the process of substrate transport (Rong et al., 2014). Also, we demonstrated that glutamate transporter GLT-1 undergoes a complicated conformational shift between TM2 and TM4 domains during transport cycle (Rong et al., 2016). There are 50 more amino acid residues in mammalian EAATs than bacterial protein in the TM4b-4c loop (Yernool et al., 2004) (Fig. 1). We introduced cysteine residues at each residue from Q189 to G245 to determine the accessibility and functionality of the TM4b-4c loop. Transport activities of 13 mutants were less than $15 \%$ and do not warrant further investigation. Aqueous accessibility of individual cysteines was determined by membrane-impermeable MTSET and membrane-permeable MTSEA. This reaction can lead to variable degrees of loss of function of the membrane protein, depending on the location of the cysteine and the importance of that position to the function of the protein. F190C, V238C, and A243C were sensitive to MTSET (Fig. 3A). We also observed that MTSEA inhibited the transport activity of Q189C, F190C, V238C, A243C, and L244C (Fig. 3B). This result, coupled with our findings on the membrane-impermeant sulfhydryl reagent reactivity of other cysteine-substituted residues suggested that Q189 and L244 are located in an aqueous pore within the membrane, whereas F190, V238, and A243 may be located around the extracellular side of the plasma membrane. It was suggested that MTSEA approaches Q189C and L244C cysteine residues from the cytoplasm. Alternatively, F190, V238, and A243 may be situated in the transport pathway, which is exposed to the aqueous environment. Additionally, the transport activity inhibition of Q189C with MTSEA is the highest compared with the other residues, indicating that this residue is quite accessible.

Intersubunit distance measurements showed that the TM4b-4c loop is situated on the outer circumference of EAAT1. Koch and others have suggested that it may form a loop that extends from the entrance hall to the outer edge of EAATs and back to the core (Koch et al., 2007b). It was also postulated that 

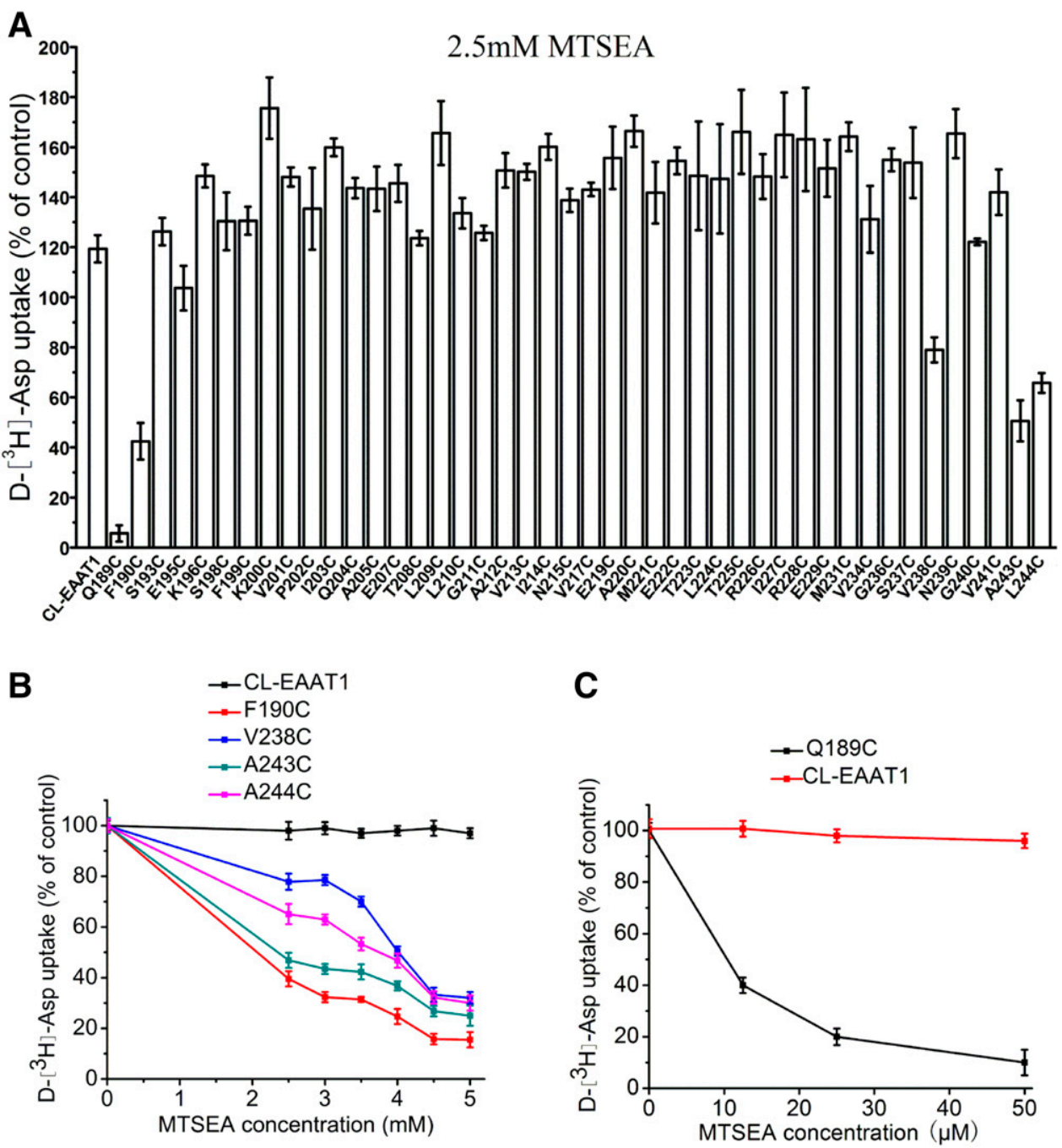

Fig. 4. Effect of MTSEA on uptake activity of single cysteine mutants of the TM4b-4c loop of CL-EAAT1. HeLa cells expressing single cysteine mutants or CL-EAAT1 were preincubated in $\mathrm{NaCl}$-containing medium with MTSEA for 5 minutes at room temperature, washed twice with ChCl-containing solution, and subsequently D- $\left[{ }^{3} \mathrm{H}\right]$-aspartate transport was assayed. Data represent the percentage of the remaining uptake activity after incubation with MTSEA relative to values obtained in the absence of MTSEA and represent the mean \pm S.E. of at least three different experiments performed in triplicate. (A) Effect of MTSEA on transport activity of single cysteine mutants of TM4b-4c loop of CL-EAAT1. The concentration of MTSEA used in this study was $2.5 \mathrm{mM}$. (B and C) Effect of different concentrations of MTSEA on transport activity of Q189C, F190C, V238C, A243C, and L244C. the TM4b-4c loop may exert effects on the coordination of the cooperativity of the three subunits for the binding of $\mathrm{Na}^{+}$ions (Koch et al., 2007b). A previous study postulated that the TM4b-4c loop may be located on the extracellular vestibule of glutamate transporters (Yernool et al., 2004). In addition, the TM4b-4c loop of EAATs have previously been speculated to be accessible to the extracellular space (Pines et al., 1992; Grunewald et al., 1998; Seal et al., 2000). Our aqueous accessibility data indicated that the TM4b-4c loop may not simply be situated on the extracellular side of the membrane. It may form multiple reentrant membrane loops at the cell surface that lead to the MTSET modification of F190C, V238C, and A243C. Additionally, the results of MTS modification suggest a nonperiodic secondary structure.

Five single cysteine mutants, Q189C, F190C, V238C, A243C, and $\mathrm{L} 244 \mathrm{C}$, whose mutated residues are roughly situated at

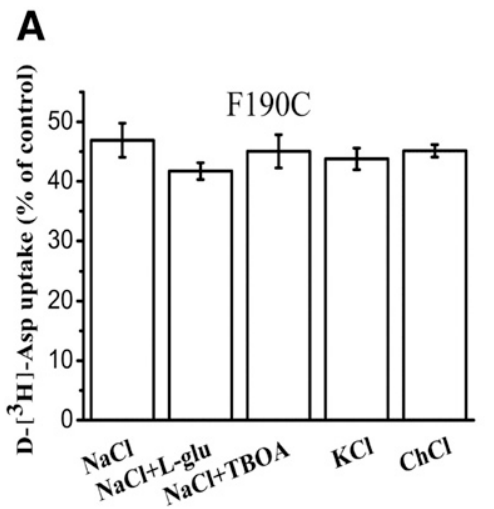

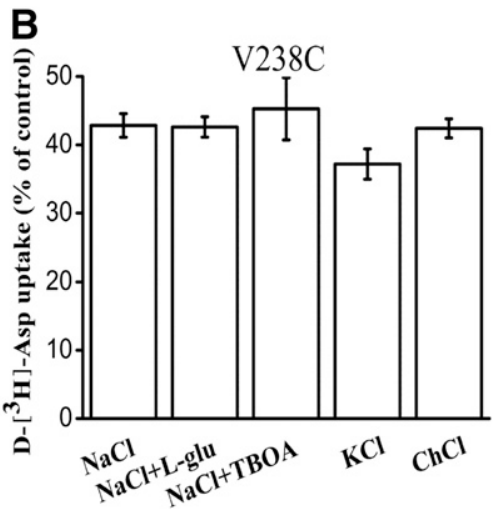

Fig. 5. Effect of external media on the inhibition of transport activity by MTSET. HeLa cells expressing F190C (A) and V238C (B) were preincubated for 5 minutes in the presence or absence of $1.5 \mathrm{mM}$ (A) and $1.0 \mathrm{mM}$ (B) MTSET. The indicated preincubation solutions contained $\mathrm{NaCl}$ solution, $\mathrm{NaCl}$ solution $+1 \mathrm{mM}$ L-glutamate, $\mathrm{NaCl}$ solution $+20 \mu \mathrm{M}$ TBOA, $\mathrm{KCl}$ solution, and ChCl solution. After washing, D- $\left[{ }^{3} \mathrm{H}\right]$-aspartate uptake was measured. The results represent the mean \pm S.E. (error bars) of at least three experiments performed in triplicate and are given as a percentage of the transport activity of samples preincubated in the same medium without MTSET $(* P<0.05)$. 

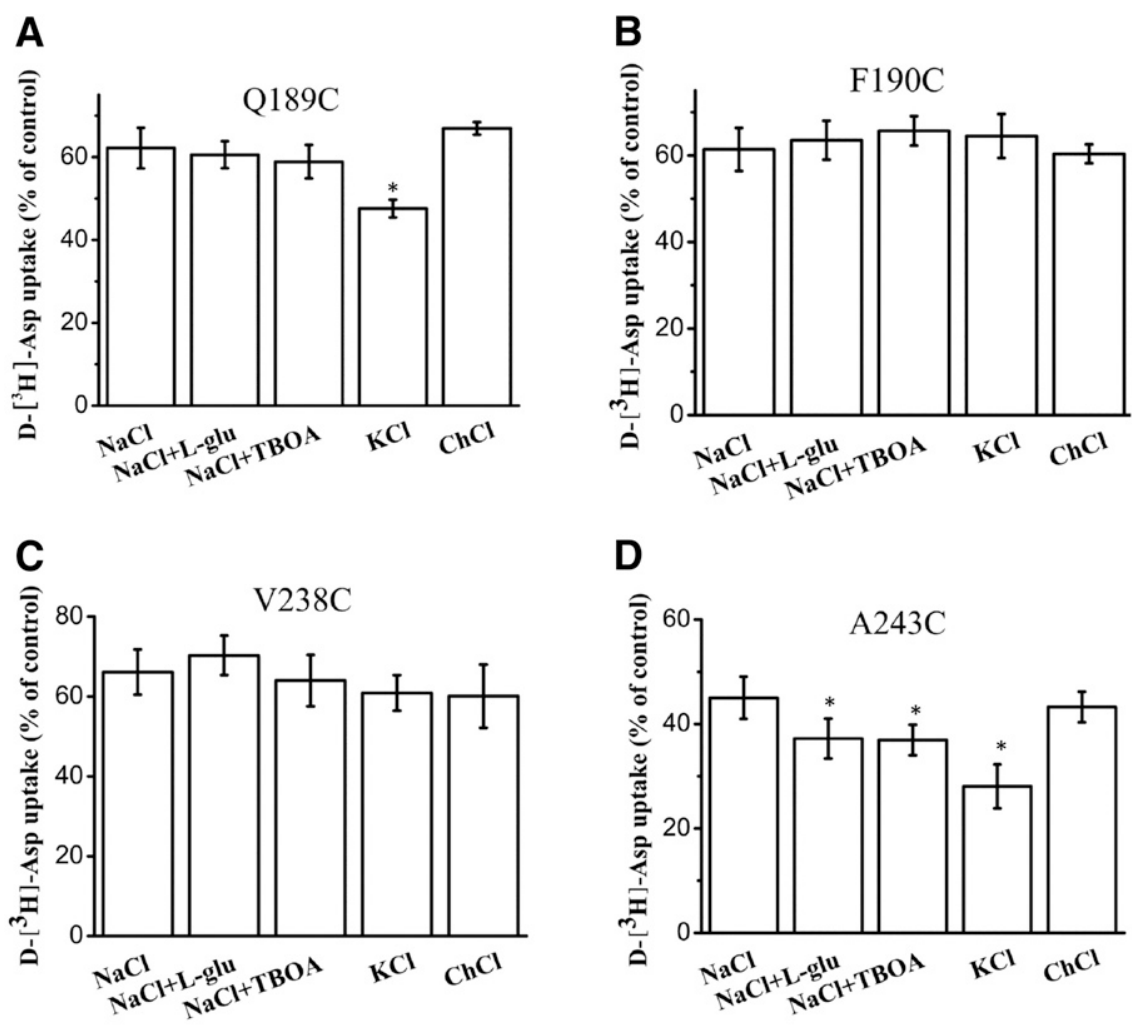

Fig. 6. Effect of external media on the inhibition of transport activity by MTSEA. HeLa cells expressing Q189C (A), F190C (B), V238C (C), A243C (D), and L244C (E) were preincubated for 5 minutes in the presence or absence of $10 \mu \mathrm{M}(\mathrm{A}), 1.5 \mathrm{mM}$ (B), $3.5 \mathrm{mM}$ (C), $2.5 \mathrm{mM}(\mathrm{D})$, and $3.5 \mathrm{mM}$ (E) MTSEA. The indicated preincubation solutions contained $\mathrm{NaCl}$ solution, $\mathrm{NaCl}$ solution $+1 \mathrm{mM}$ L-glutamate, $\mathrm{NaCl}$ solution $+20 \mu \mathrm{M}$ TBOA, $\mathrm{KCl}$ solution, and $\mathrm{ChCl}$ solution. After washing, $\mathrm{D}-\left[{ }^{3} \mathrm{H}\right]$-aspartate uptake was measured. Results represent the mean \pm S.E. (error bars) of at least three experiments performed in triplicate and are given as a percentage of the transport activity of samples preincubated in the same medium without MTSEA $(* P<0.05)$.

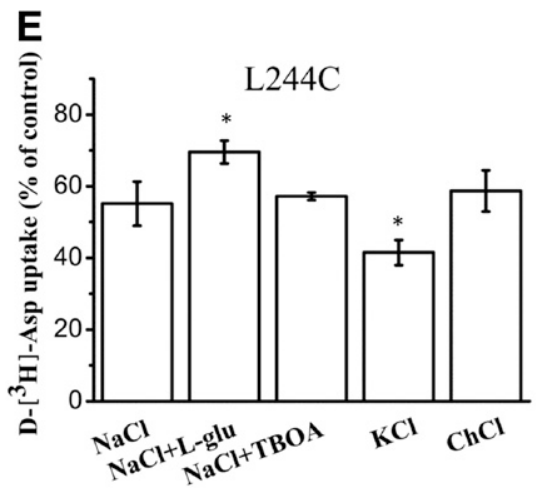

opposite ends of the TM4b-4c loop, are sensitive to the sulfhydryl compounds MTSET or MTSEA. To maximize the chances of sulfhydryl modification of these mutants from either side of the membrane, the cells were exposed to the sulfhydryl reagent in the presence of different external media. DL-TBOA, a nontransported and competitive inhibitor of EAAT1, is expected to increase the percentage of outwardfacing transporters (Boudker et al., 2007). Conversely, the binding of L-glutamate may block access of the sulfhydryl reagents to the cysteine side chain. It is expected that potassium and glutamate will increase the percentage of inward-facing transporters (Bergles et al., 2002; Reyes et al., 2009). The explanation for the increased inhibition of transport by MTSEA in the presence of potassium is that when the transporter becomes inward facing, mutants Q189C, A243C, and L244C may move and become more accessible (Fig. 6, A, D, and E). However, L-glutamate protects the L244C from modification by MTSEA (Fig. 6E). For A243C, glutamate, potassium, and DL-TBOA each augmented the transporter activity inhibition by MTSEA (Fig. 6D). It may be explained that A243C accessibility increased in both outward-facing and inward-facing transporters during the transport cycle. Our previous results have demonstrated that $\mathrm{A} 243 \mathrm{C}$ is accessible to MTSET, and inhibition was augmented in the presence of DL-TBOA (Rong et al., 2016). Our current results show that the inhibition of uptake activity of A243C by MTSEA is augmented in the presence of potassium and L-glutamate, but inhibition by MTSET is unaffected by these compounds. Presumably, MTSEA may be able to reach the cysteine at position A243C from both the outside and the inside of the cell membrane. F190C and V238C were not impacted by MTSET or MTSEA in the presence of TBOA or potassium and glutamate (Fig. 4, A and B; Fig. 5, A and B). Thus, for cysteine mutants $\mathrm{F} 190 \mathrm{C}$ and $\mathrm{V} 238 \mathrm{C}$, this reactivity is not conformationally sensitive, suggesting that they are always accessible to the extracellular side or transport pathway. To investigate whether the loss of uptake activity detected 
A

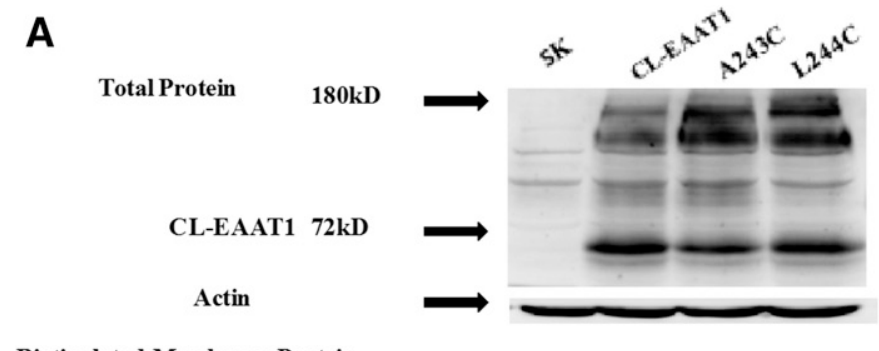

Biotinylated Membrane Protein

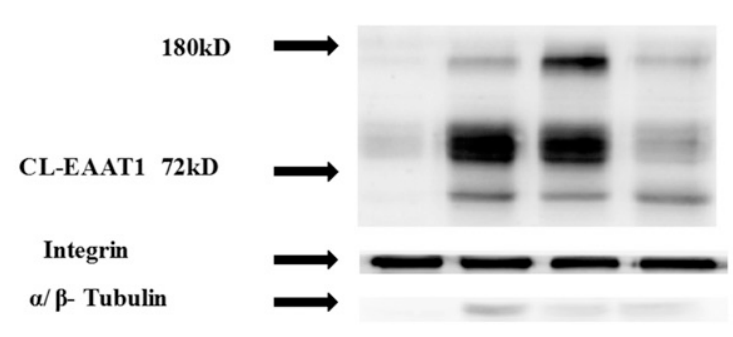

Non-biotinylated protein
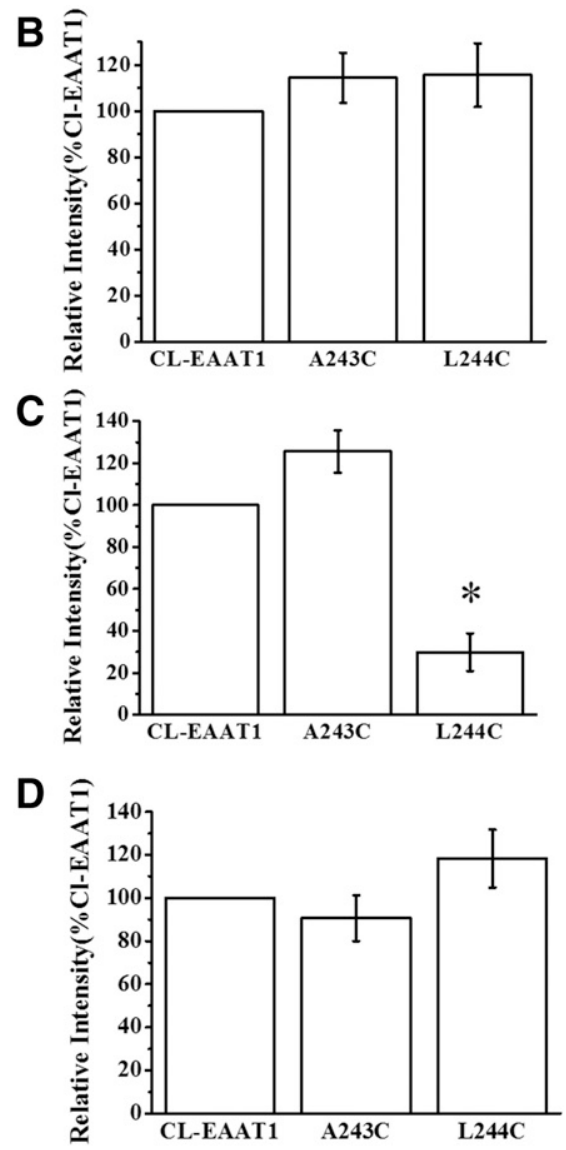

E

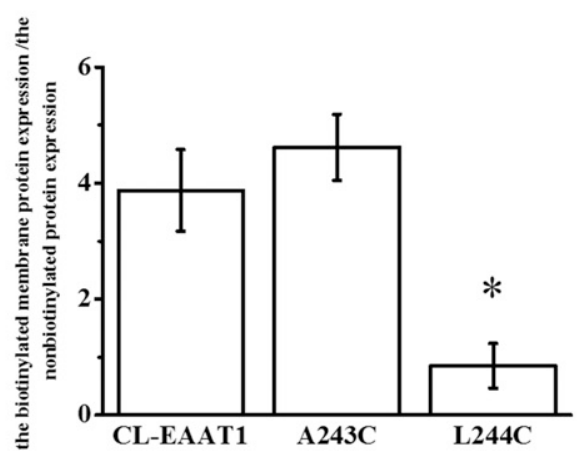

$\mathbf{F}$

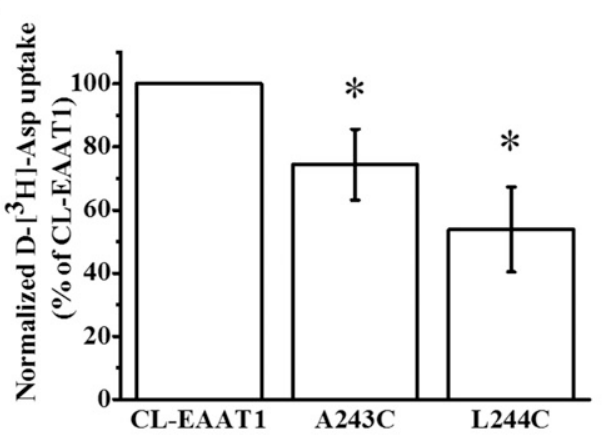

Fig. 7. Membrane expression of V243C and V244C mutants. HeLa cells were transfected with the CL-EAAT1, V243C, and V244C mutants. (A) The total proteins, biotinylated membrane proteins, and nonbiotinylated proteins were measured by Western blot as described in Materials and Methods. Blots of all proteins were probed with the anti-EAAT1 antibody. Each blot of the biotinylated membrane proteins was probed for the internal plasma membrane marker integrin and the absence of $\alpha / \beta$-tubulin, an endogenous cytosolic protein representing the negative control in the biotinylated membrane proteins. Each blot of the nonbiotinylated proteins was probed for the presence of $\alpha / \beta$-tubulin. Each blot of the total proteins was probed for the presence of actin. (B) Densitometric analysis of the total proteins for each mutant normalized to internal marker (actin) and represented as a percentage of CL-EAAT1. (C) Densitometric analysis of the biotinylated membrane proteins for each mutant normalized to an internal marker (integrin) and represented as a percentage of CL-EAAT1. (D) Densitometric analysis of the nonbiotinylated proteins for each mutant normalized to an internal marker $(\alpha / \beta$-tubulin) and represented as a percentage of CL-EAAT1. (E) The ratio of the biotinylated membrane protein expression and nonbiotinylated protein expression of CL-EAAT1 and mutants. (F) D- $\left[{ }^{3} \mathrm{H}\right]$-Asp uptake activity normalized to relative cell surface expression. Values represent the mean \pm S.E. of at least three different experiments performed in triplicate. Values significantly different from those of CL-EAAT1 were determined by one-way analysis of variance $(* P<0.05)$.

with $\mathrm{A} 243 \mathrm{C}$ and $\mathrm{L} 244 \mathrm{C}$ was owing to an absence of the transporter at the cell surface or whether these have intrinsic transport defects, the levels of the mutant proteins in the plasma membrane were evaluated by cell surface biotinylation. The decreased transport activity of L244C can be partially explained by the inability of the transporter to traffic or insert into the plasma membrane because of its absence from the biotinylated fraction (Fig. 7, C and E). A243C showed about 90\% activity compared with CL-EAAT1. Uptake activity normalized to relative cell surface expression shows that A243C and L244C have an intrinsic transport defect (Fig. 7F).

Previous studies suggest state-dependent trypsin cleavage sites and conformational changes have been found between 
TM3 and TM4 on GLT-1 (Grunewald and Kanner, 1995; Bergles et al., 2002). This is consistent with our data. Conversely, fluorescence resonance energy transfer (FRET) results suggested little conformational change of the TM4b-4c loop during the transport cycle (Koch and Larsson, 2005). However, FRET measurements were the mean FRET efficiency, and they cannot uncover true complex conformational changes during the glutamate transport cycle. Large-scale transport domain movements were observed by single-molecule FRET imaging in a bacterial homolog of glutamate transporters (Akyuz et al., 2013). Therefore, FRET measurements may not accurately predict movement of the TM4b-4c loop. A previous study postulated that the TM4b-4c loop may be located in the extracellular vestibule of eukaryotic EAATs (Yernool et al., 2004), but this hypothesis is inconsistent with our results. $N$-Glycosylation sites in the TM4b-4c loop suggest these may play an important role in the post-translational processing of these proteins (Canul-Tec et al., 2017).

From the discussion above, we suggest that the majority of the residues in the TM4b-4c loop may form multiple reentrant membrane loops. Q189C, A243C, and L244C of the TM4b-4c loop are conformationally sensitive and may have roles in the transport pathway during the transport cycle. The function of other residues of the TM4b-4c loop is not clear. The TM4b-4c loop may be located in the periphery of the transporter, based on the SWISS-MODEL (Fig. 8). Additionally, the result of the MTS experiment combined with the SWISS-MODEL suggested that some residues of the TM4b-4c loop may be positioned at the transport domain of the protein.

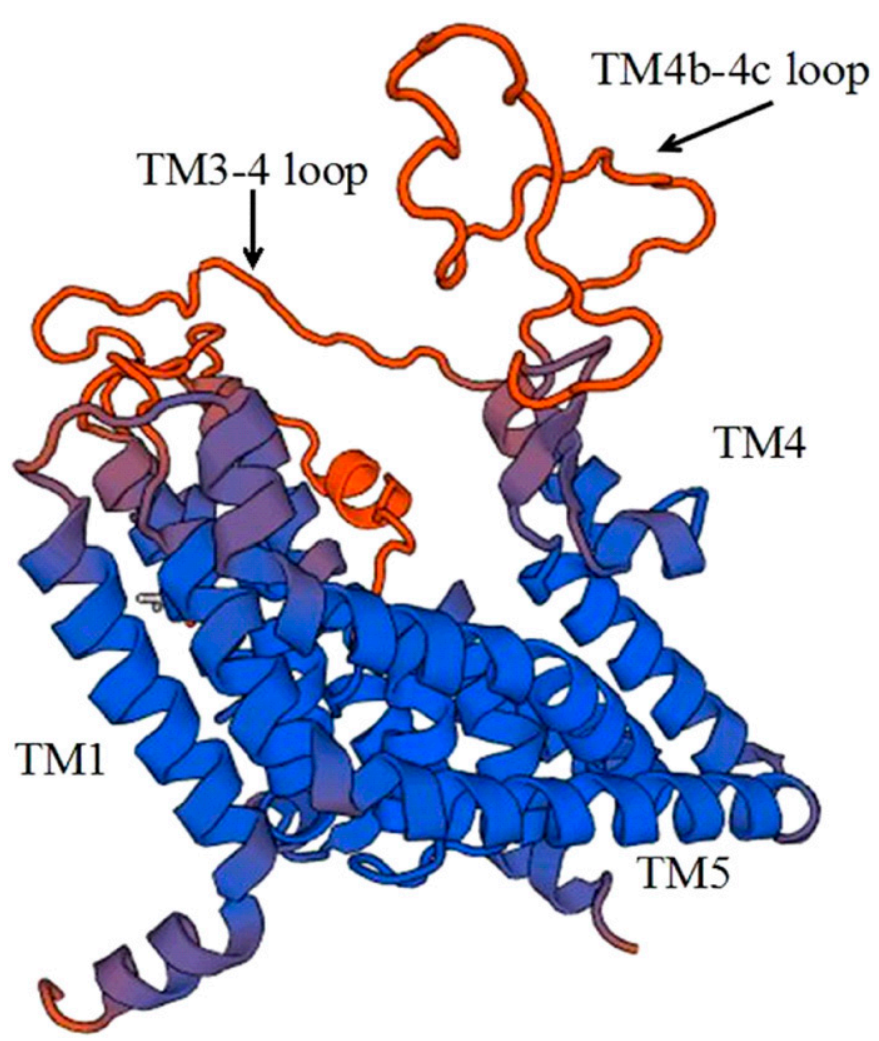

Fig. 8. Structure model from 5llm.1.A. Three-dimensional image of EAAT1 was generated by the SWISS-MODEL homology modeling. Arrows depict the TM3-4 and TM4b-4c loops (Arnold et al., 2006; Kiefer et al., 2009; Biasini et al., 2014).

\section{Authorship Contributions}

Participated in research design: $\mathrm{Qu}$.

Conducted experiments: W. Zhang, X. Zhang.

Performed data analysis: W. Zhang, X. Zhang, Qu.

Wrote or contributed to the writing of the manuscript: W. Zhang, $\mathrm{X}$. Zhang, Qu.

\section{References}

Akyuz N, Altman RB, Blanchard SC, and Boudker O (2013) Transport dynamics in a glutamate transporter homologue. Nature 502:114-118.

Arnold K, Bordoli L, Kopp J, and Schwede T (2006) The Swiss-model workspace: a web-based environment for protein structure homology modelling. Bioinformatics 22:195-201.

Arriza JL, Eliasof S, Kavanaugh MP, and Amara SG (1997) Excitatory amino acid transporter 5, a retinal glutamate transporter coupled to a chloride conductance. Proc Natl Acad Sci USA 94:4155-4160.

Arriza JL, Fairman WA, Wadiche JI, Murdoch GH, Kavanaugh MP, and Amara SG (1994) Functional comparisons of three glutamate transporter subtypes cloned from human motor cortex. J Neurosci 14:5559-5569.

Bergles DE, Tzingounis AV, and Jahr CE (2002) Comparison of coupled and uncoupled currents during glutamate uptake by GLT-1 transporters. J Neurosci 22 10153-10162.

Biasini M, Bienert S, Waterhouse A, Arnold K, Studer G, Schmidt T, Kiefer F, Gallo Cassarino T, Bertoni M, Bordoli L, et al. (2014) Swiss-model: modelling protein tertiary and quaternary structure using evolutionary information. Nucleic Acids Res 42:W252-W258.

Boudker O, Ryan RM, Yernool D, Shimamoto K, and Gouaux E (2007) Coupling substrate and ion binding to extracellular gate of a sodium-dependent aspartate transporter. Nature 445:387-393.

Brew H and Attwell D (1987) Electrogenic glutamate uptake is a major current carrier in the membrane of axolotl retinal glial cells. Nature 327:707-709.

Canul-Tec JC, Assal R, Cirri E, Legrand P, Brier S, Chamot-Rooke J, and Reyes N (2017) Structure and allosteric inhibition of excitatory amino acid transporter 1. Nature 544:446-451.

Crisman TJ, Qu S, Kanner BI, and Forrest LR (2009) Inward-facing conformation of glutamate transporters as revealed by their inverted-topology structural repeats. Proc Natl Acad Sci USA 106:20752-20757.

Diamond JS and Jahr CE (1997) Transporters buffer synaptically released glutamate on a submillisecond time scale. J Neurosci 17:4672-4687.

Fairman WA, Vandenberg RJ, Arriza JL, Kavanaugh MP, and Amara SG (1995) An excitatory amino-acid transporter with properties of a ligand-gated chloride channel. Nature 375:599-603.

Fuerst TR, Niles EG, Studier FW, and Moss B (1986) Eukaryotic transientexpression system based on recombinant vaccinia virus that synthesizes bacteriophage T7 RNA polymerase. Proc Natl Acad Sci USA 83:8122-8126.

Grewer C, Balani P, Weidenfeller C, Bartusel T, Tao Z, and Rauen T (2005) Individual subunits of the glutamate transporter EAAC1 homotrimer function independently of each other. Biochemistry 44:11913-11923.

Grunewald M and Kanner B (1995) Conformational changes monitored on the glutamate transporter GLT-1 indicate the existence of two neurotransmitter-bound states. J Biol Chem 270:17017-17024.

Grunewald M, Bendahan A, and Kanner BI (1998) Biotinylation of single cysteine mutants of the glutamate transporter GLT-1 from rat brain reveals its unusual topology. Neuron 21:623-632.

Huang Z and Tajkhorshid E (2008) Dynamics of the extracellular gate and ionsubstrate coupling in the glutamate transporter. Biophys $J$ 95:2292-2300.

Hussainzada N, Banerjee A, and Swaan PW (2006) Transmembrane domain VII of the human apical sodium-dependent bile acid transporter ASBT (SLC10A2) lines the substrate translocation pathway. Mol Pharmacol 70:1565-1574.

Hussainzada N, Khandewal A, and Swaan PW (2008) Conformational flexibility of helix VI is essential for substrate permeation of the human apical sodiumdependent bile acid transporter. Mol Pharmacol 73:305-313.

Kanai Y and Hediger MA (1992) Primary structure and functional characterization of a high-affinity glutamate transporter. Nature 360:467-471.

Kanner BI (2007) Gate movements in glutamate transporters. ACS Chem Biol 2: 163-166.

Kanner BI and Schuldiner S (1987) Mechanism of transport and storage of neurotransmitters. CRC Crit Rev Biochem 22:1-38.

Kanner BI and Sharon I (1978) Active transport of L-glutamate by membrane vesicles isolated from rat brain. Biochemistry 17:3949-3953.

Kiefer F, Arnold K, Künzli M, Bordoli L, and Schwede T (2009) The Swiss-model repository and associated resources. Nucleic Acids Res 37:D387-D392.

Koch HP, Brown RL, and Larsson HP (2007a) The glutamate-activated anion conductance in excitatory amino acid transporters is gated independently by the individual subunits. J Neurosci 27:2943-2947.

Koch HP, Hubbard JM, and Larsson HP (2007b) Voltage-independent sodiumbinding events reported by the 4B-4C loop in the human glutamate transporter excitatory amino acid transporter 3. J Biol Chem 282:24547-24553.

Koch HP and Larsson HP (2005) Small-scale molecular motions accomplish glutamate uptake in human glutamate transporters. J Neurosci 25:1730-1736.

Leary GP, Stone EF, Holley DC, and Kavanaugh MP (2007) The glutamate and chloride permeation pathways are colocalized in individual neuronal glutamate transporter subunits. J Neurosci 27:2938-2942.

Levy LM, Warr O, and Attwell D (1998) Stoichiometry of the glial glutamate transporter GLT-1 expressed inducibly in a Chinese hamster ovary cell line selected for low endogenous $\mathrm{Na}+$-dependent glutamate uptake. $J$ Neurosci 18: 9620-9628. 
Nicholls D and Attwell D (1990) The release and uptake of excitatory amino acids. Trends Pharmacol Sci 11:462-468.

Otis TS, Wu YC, and Trussell LO (1996) Delayed clearance of transmitter and the role of glutamate transporters at synapses with multiple release sites. $J$ Neurosci 16:1634-1644. Owe SG, Marcaggi P, and Attwell D (2006) The ionic stoichiometry of the GLAST glutamate transporter in salamander retinal glia. J Physiol 577:591-599.

Pines G, Danbolt NC, Bjørås M, Zhang Y, Bendahan A, Eide L, Koepsell H, StormMathisen J, Seeberg E, and Kanner BI (1992) Cloning and expression of a rat brain L-glutamate transporter. Nature 360:464-467.

Reyes N, Ginter C, and Boudker O (2009) Transport mechanism of a bacterial homologue of glutamate transporters. Nature 462:880-885.

Rong X, Tan F, Wu X, Zhang X, Lu L, Zou X, and Qu S (2016) TM4 of the glutamate transporter GLT-1 experiences substrate-induced motion during the transport cycle. Sci Rep 6:34522.

Rong X, Zomot E, Zhang X, and Qu S (2014) Investigating substrate-induced motion between the scaffold and transport domains in the glutamate transporter EAAT1. Mol Pharmacol 86:657-664.

Rothstein JD, Dykes-Hoberg M, Pardo CA, Bristol LA, Jin L, Kuncl RW, Kanai Y, Hediger MA, Wang Y, Schielke JP, et al. (1996) Knockout of glutamate transporters reveals a major role for astroglial transport in excitotoxicity and clearance of glutamate. Neuron 16:675-686.

Seal RP and Amara SG (1998) A reentrant loop domain in the glutamate carrier EAAT1 participates in substrate binding and translocation. Neuron 21:1487-1498.

Seal RP, Leighton BH, and Amara SG (2000) A model for the topology of excitatory amino acid transporters determined by the extracellular accessibility of substituted cysteines. Neuron 25:695-706.

Shrivastava IH, Jiang J, Amara SG, and Bahar I (2008) Time-resolved mechanism of extracellular gate opening and substrate binding in a glutamate transporter. $J$ Bio Chem 283:28680-28690.
Storck T, Schulte S, Hofmann K, and Stoffel W (1992) Structure, expression, and functional analysis of a $\mathrm{Na}(+)$-dependent glutamate/aspartate transporter from rat brain. Proc Natl Acad Sci USA 89:10955-10959.

Tanaka K, Watase K, Manabe T, Yamada K, Watanabe M, Takahashi K, Iwama H, Nishikawa T, Ichihara N, Kikuchi T, et al. (1997) Epilepsy and exacerbation of brain injury in mice lacking the glutamate transporter GLT-1. Science 276: 1699-1702.

Teichman S, Qu S, and Kanner BI (2009) The equivalent of a thallium binding residue from an archeal homolog controls cation interactions in brain glutamate transporters. Proc Natl Acad Sci USA 106:14297-14302.

Tong G and Jahr CE (1994) Block of glutamate transporters potentiates postsynaptic excitation. Neuron 13:1195-1203.

Verdon G and Boudker O (2012) Crystal structure of an asymmetric trimer of a bacterial glutamate transporter homolog. Nat Struct Mol Biol 19:355-357.

Verdon G, Oh S, Serio RN, and Boudker O (2014) Coupled ion binding and structural transitions along the transport cycle of glutamate transporters. eLife 3:e02283.

Wadiche JI, Arriza JL, Amara SG, and Kavanaugh MP (1995) Kinetics of a human glutamate transporter. Neuron 14:1019-1027.

Yernool D, Boudker O, Jin Y, and Gouaux E (2004) Structure of a glutamate transporter homologue from Pyrococcus horikoshii. Nature 431:811-818.

Zerangue N and Kavanaugh MP (1996) Flux coupling in a neuronal glutamate transporter. Nature 383:634-637.

Address correspondence to: Shaogang Qu, Clinical Medicine Research Centre, Shunde Hospital, Southern Medical University, Foshan, Guangdong 528300, China. E-mail: sgq9528@163.com 